\title{
Desenvolvimento de casos clínicos para o ensino do raciocínio diagnóstico*
}

Developing clinical cases to reach diagnostic reasoning

Desarrollo de casos clínicos para la enseñanza del razonamiento diagnóstico

Fernanda dos Santos Nogueira Góes ${ }^{1}$, Maria Célia Barcellos Dalri², Luciana Mara Monti Fonseca ${ }^{3}$, Silvia Rita Marin da Silva Canini ${ }^{4}$, Carmen Gracinda Silvan Scochi ${ }^{5}$

* Extraído da tese intitulada "Desenvolvimento e avaliação de objeto virtual de aprendizagem interativo sobre o raciocínio diagnóstico em enfermagem aplicado ao recém-nascido pré-termo", apresentada ao Programa de Pós-Graduação Enfermagem em Saúde Pública da Escola de Enfermagem de Ribeirão Preto da Universidade de São Paulo, no ano de 2010.

${ }^{1}$ Enfermeira, Doutora Enfermagem em Saúde Pública. Professora Doutora da Escola de Enfermagem de Ribeirão Preto da Universidade de São Paulo (EERP/USP). Ribeirão Preto, São Paulo, Brasil. E-mail: fersngoes@eerp.usp.br.

2 Enfermeira, Doutora em Enfermagem Fundamental. Professora Associada da EERP/USP. Ribeirão Preto, São Paulo, Brasil. E-mail: macdalri@eerp.usp.br.

3 Enfermeira, Doutora em Saúde Pública. Professora Doutora da EERP/USP. Ribeirão Preto, São Paulo, Brasil. E-mail: lumonti@eerp.usp.br.

${ }^{4}$ Enfermeira, Doutora em Enfermagem. Professora Associada da EERP/USP. Ribeirão Preto, São Paulo, Brasil. E-mail: canini@eerp.usp.br.

${ }^{5}$ Enfermeira, Livre-Docente. Professora Titular da EERP/USP. Bolsista Produtividade em Pesquisa 1 B do CNPq. Coordenadora da Área de Enfermagem na CAPES. Ribeirão Preto, São Paulo, Brasil. E-mail: cscochi@eerp.usp.br.

\section{RESUMO}

Objetiva-se relatar a elaboração e validação de estudos de caso em enfermagem neonatal adaptados segundo referencial educacional específico. Estudo metodológico desenvolvido em hospital público do estado de São Paulo. Coletaram-se dados de 22 recém-nascidos (15 - verificação de confiabilidade na coleta dos casos), dos quais sete foram adaptados em estudos de caso a partir de seis passos: seleção dos recursos e métodos, objetivos, complexidade, validação das evidências, roteiros e validade do conteúdo. Os objetivos dos casos contemplam o ensino, assistência e qualidade técnica do caso. Sobre a complexidade dos casos, optou-se por elaborá-los considerando as necessidades humanas básicas, raciocínio diagnóstico de Risner e taxonomia II da NANDA-I. A elaboração e validação dos casos a partir de referencial específico colaborou para que os estudos de casos possam se tornar recurso importante na aprendizagem. Percebeu-se lacuna de conhecimento no que se refere à validação de estudos de caso para o ensino.

Descritores: Estudo de Caso; Tecnologia Educacional; Aprendizagem; Educação em Enfermagem.

\section{ABSTRACT}

The objective of this study is to report the creation and validation of case studies in neonatal nursing adapted according to a specific educational framework. This methodological study was performed in a public hospital in the state of São Paulo. Data were collected with 22 newborns (15 - verification of reliability in the case collection), seven of which were adapted in case studies following six steps: resource and method selection, goals, complexity, validation of the evidence, scripts and content validity. The cases' goals comprise teaching, care and the technical quality of the case. Regarding the complexity of cases, they were elaborated considering the basic human needs, Risner's diagnostic reasoning, and NANDA-I taxonomy II. The creation and validation of cases based on a specific framework contributed to case studies becoming an important learning resource. A knowledge gap was observed regarding the validation of case studies for teaching.

Descriptors: Case Studies; Educational Technology; Learning; Education, Nursing.

\section{RESUMEN}

Se objetiva informar la elaboración y validación de estudios de caso en enfermería neonatal, adaptados según referencial educativo específico. Estudio metodológico, desarrollado en hospital público del estado de São Paulo. Se recolectaron datos de 22 recién nacidos (15- verificación de confiabilidad en recolección de casos); de ellos, siete fueron adaptados a estudios de caso siguiéndose seis pasos: selección de recursos y métodos, objetivos, complejidad, validación de evidencias, rutinas y validad del contenido. Los objetivos de los casos contemplan enseñanza, atención y calidad técnica del caso. Sobre complejidad de los casos, se optó por elaborarlos considerando las necesidades humanas básicas, razonamiento diagnóstico de Risner y taxonomía II de NANDA-I. La elaboración y validación de casos a partir de referencial específico ayudó a que los estudios de caso puedan convertirse en recurso importante de aprendizaje. Se percibió vacío de conocimientos en referencia a validación de estudios de caso para enseñanza.

Descriptores: Estudios de Casos; Tecnología Educacional; Aprendizaje; Educación en Enfermería. 


\section{INTRODUÇÃO}

As mudanças no sistema de saúde brasileiro, as exigências pelo cuidado seguro ao paciente, família e comunidade têm evidenciado a importância da formação inicial e permanente de enfermeiros. Transformações econômicas e sociais desafiam o setor educacional na busca de ensino que estimule a reflexão e criticidade dos estudantes $^{(1)}$.

Contudo, no ensino de enfermagem neonatal, muitos conceitos e técnicas são difíceis de serem apreendidos pelos alunos, pois, muitas vezes a assistência direta ao recém-nascido não é possível, especialmente em situações que a instabilidade clínica está presente, podendo dificultar o estímulo do pensamento crítico $^{(2-4)}$, e a aquisição de competências específicas da profissão.

Dessa forma, um dos desafios na educação superior em enfermagem é ensinar ${ }^{(5)}$ a realizar o raciocínio diagnóstico, mediante o emprego de habilidades cognitivas e dedutivas, de modo que o processo seja rápido, eficaz e acurado.

A aplicação do processo de enfermagem ${ }^{(2)}$ proporciona os fundamentos para as habilidades de pensamento crítico e raciocínio diagnóstico, necessárias ao exercício da profissão de uma maneira segura e eficiente.

Para que a educação em enfermagem proporcione as habilidades requeridas para o exercício da profissão, os cursos de graduação em enfermagem devem definir linguagem padronizada(5) para o ensino do raciocínio diagnóstico e defender a importância do conhecimento da ciência de enfermagem para o desenvolvimento da profissão e a segurança do paciente, família e comunidade.

Soma-se também que a desarticulação entre o valor atribuído por enfermeiros que não consideram a relevância do processo de enfermagem na sua prática diária decorre de, entre outras coisas, lacunas na formação inicial e permanente dos enfermeiros ${ }^{(5)}$.

$\mathrm{Na}$ tentativa de auxiliar na formação de enfermeiros no que tange ao ensino do raciocínio diagnóstico, o estudo de caso, enquanto estratégia de ensino, pode ser um recurso facilitador para gerar a atenção devida para sua utilização na prática clínica.

Define-se estudo de caso como análise minuciosa de uma situação real(6), que permite a dialogicidade entre professores a alunos, ou seja, a aprendizagem centrada no aluno e o professor como mediador desse processo(6-7).

Em relação ao ensino, uma das principais vantagens do estudo de caso é a abordagem orientada para perguntas e não para respostas ${ }^{(5,7)}$. A utilização de estudos de caso estimula a participação ativa dos alunos no processo ensino-aprendizagem ${ }^{(1,5-9)}$, o interesse de alunos $^{(1,6,8-9)}$, a articulação entre teoria e prática ${ }^{(3-5,8,10)}, 0$ pensamento crítico $^{(1-11)}$, a reflexão, a aprendizagem a partir de experiência, e a argumentação(7,11).

0 estudo de caso também auxilia o aluno para o estabelecimento de prioridades ${ }^{(3,10)}$, além de favorecer a avaliação das competências dos estudantes relacionadas à cognição, comunicação, procedimentos e trabalho em equipe ${ }^{(9,12-13)}$.

Outro aspecto apontado é que o ensino mediado pelo estudo de caso permite o feedback imediato para o aluno, atendendo a necessidade imediata de respostas do jovem da atualidade ${ }^{(1,13-14)}$.

A utilização de estudo de caso também pode interferir na assistência ao paciente, família e comunidade, ou seja, quando a aprendizagem ocorre primeiramente a partir de estudos de caso, há menores riscos de erros no cuidado do paciente colaborando para sua segurança ${ }^{(3,7,12)}$, pois mantêm ambiente seguro, criativo e de baixo custo de ensino.

No que se refere ao processo de enfermagem, a utilização de estudos de casos por estudantes ou enfermeiros pode auxiliá-los a melhorar suas habilidades cognitivas, pois os casos fornecem exemplos concretos dos princípios do raciocínio diagnóstico ${ }^{(4,15)}$.

Soma-se também que os estudos de caso facilitam à padronização de diagnósticos e intervenções de enfermagem para grupos específicos de clientes, atribuição de valores científicos à prática de enfermagem, durante a formação do enfermeiro alicerçada na realidade clínica ${ }^{(4,15)}$.

Apesar das vantagens do uso de casos na enfermagem, há poucas publicações relativas à adoção de um referencial teórico para a construção de casos para o ensino, a qual é considerada como relevante(7) pois o planejamento, organização e a discussão dos casos são essenciais para garantir a eficácia do processo de aprendizagem.

Assim, tem-se a intenção de relatar o processo de elaboração e validação de estudos de caso em enfermagem neonatal para os quais foram utilizados casos clínicos reais de recém-nascidos prematuros, adaptados a partir de referencial específico para construção de casos com finalidade educacional(4).

\section{MATERIAL E MÉTODO}

Estudo metodológico desenvolvido em um hospital público brasileiro no estado de São Paulo, com a finalidade 
de construir e validar casos para serem utilizados enquanto estratégia de estudo de caso.

O estudo seguiu as normas éticas nacionais e internacionais. Certificado de Apresentação para Apreciação Ética (CAAE) 0193.0.004.000-08.

\section{Sujeitos do estudo}

Para elaboração de estudos de caso de recémnascidos prematuros (RNPT) para serem aplicados no ensino de enfermagem neonatal fez-se a opção por coletar dados reais de prematuros hospitalizados em uma Unidade de Cuidados Intermediários Neonatal (UCIN) de um hospital público brasileiro. Foram incluídos RNPT com menos de 37 semanas de idade gestacional ao nascimento, internados em UCIN e que estavam clinicamente estáveis no momento da coleta de dados. Forma excluídos os RNPT com instabilidade hemodinâmica nas 24 horas que antecederam a coleta de dados.

Coletaram-se dados de 22 RNPT, após consentimento informado dos pais, dos quais 15 foram utilizados para verificação da confiabilidade da pesquisadora e sete para serem adaptados em estudos de caso com finalidade educacional.

Para validação das evidências coletadas e dos estudos de caso adaptados foram convidadas cinco enfermeiras atendendo às recomendações do Sistema de Pontuação para seleção de expertos adaptado(16).

Justifica-se a utilização de sistema de pontuação para seleção de expertos adaptado(16) pois, em estudo sobre validação do diagnóstico de enfermagem(17), houve dificuldades na seleção de expertos de acordo com o modelo de validação por sistema de Pontuação(18), pois haviam poucos enfermeiros com titulação. Partindo desta limitação e da experiência profissional, optou-se por elaborar novo instrumento de pontuação para seleção de expertos, adaptando as recomendações ${ }^{(16)}$.

Assim, para inclusão de experto estabeleceu-se pontuação mínima de quatro pontos; em relação à titulação computou-se apenas um ponto, mesmo que o perito possuísse todos os títulos.

Quadro 1: Instrumento de pontuação adaptado para seleção de enfermeiros em enfermagem neonatal. Ribeirão Preto, SP, Brasil, 2010.

\begin{tabular}{|c|c|c|}
\hline Categorização & Itens & Pontuação \\
\hline \multirow{4}{*}{ Titulação } & Mestre em enfermagem & 01 \\
\hline & Doutor em enfermagem & 01 \\
\hline & $\begin{array}{c}\begin{array}{c}\text { Especialista em área clínica sobre diagnóstico de } \\
\text { enfermagem }\end{array}\end{array}$ & 01 \\
\hline & $\begin{array}{l}\text { Especialista em área sobre assistência ao prematuro e à } \\
\text { família em unidade neonatal }\end{array}$ & 01 \\
\hline \multirow{2}{*}{$\begin{array}{c}\text { Experiência } \\
\text { Profissional/Assistencial }\end{array}$} & $\begin{array}{l}\text { Diagnóstico de enfermagem } \\
\end{array}$ & 02 \\
\hline & Prematuro e à família em unidade neonatal & 02 \\
\hline \multirow{2}{*}{ Ensino } & Diagnóstico de enfermagem & 02 \\
\hline & Cuidados de enfermagem ao prematuro e à família & 02 \\
\hline \multirow[b]{2}{*}{$\begin{array}{c}\text { Desenvolvimento/Orientação de } \\
\text { pesquisa }\end{array}$} & $\begin{array}{l}\text { Diagnóstico de enfermagem, vinculada ou não a trabalho de } \\
\text { conclusão de curso/dissertação/tese }\end{array}$ & 02 \\
\hline & $\begin{array}{l}\text { Cuidados de enfermagem ao prematuro e à família, } \\
\text { vinculada ou não a trabalho de conclusão de curso/ } \\
\text { dissertação/tese }\end{array}$ & 02 \\
\hline Publicação & $\begin{array}{l}\text { Artigo, livro ou capítulo sobre cuidados de enfermagem ao } \\
\text { prematuro e à família }\end{array}$ & 02 \\
\hline
\end{tabular}

\section{Coleta de dados}

Para testar a confiabilidade da coleta de dados dos RNPT pela pesquisadora do estudo optou-se por realizar, primeiramente, a calibração da pesquisadora por meio da comparação dos dados coletados em conjunto com uma enfermeira experta na linguagem padronizada dos diagnósticos de enfermagem e no cuidado ao RNPT.

A seleção da enfermeira experta ocorreu de acordo com o instrumento de pontuação para seleção de expertos

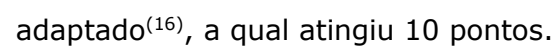

Para verificação de confiabilidade (CO) entre as avaliadoras (pesquisadora e enfermeira experta) foram processados o total de concordâncias (C) dividido pela somatória do total de concordâncias $(C)$ e discordâncias (DC): $\mathrm{CO}=\mathrm{C} / \mathrm{C}+\mathrm{DC}$.

Estabeleceu-se concordância mínima de 80\%, conforme recomendação e critério adotado em estudo anterior $^{(19)}$.

Após conclusão dessa etapa, o processo de coleta, adequação e validação dos casos clínicos foi realizado exclusivamente pela pesquisadora.

Vislumbrou-se a necessidade de que os casos clínicos refletissem os propósitos para os quais foram planejados e, assim, fez-se necessário construí-los de forma a 
atender as diretrizes para desenvolvimento de casos válidos e confiáveis.

Para adequação dos sete casos clínicos coletados seguiu-se a recomendação ${ }^{(4)}$, a qual sugere seis passos para elaboração de estudos de casos com finalidades educacionais, a saber: seleção dos recursos e métodos, objetivos dos casos, complexidade, validação das evidências, roteiros de orientação e validade do conteúdo.

De acordo com o primeiro passo, selecionou-se os recursos e métodos que foram utilizados na construção de casos.

No segundo passo, estabeleceu-se os objetivos a serem alcançados nos estudos de casos.

Como orientação do terceiro passo, decidiu-se a complexidade dos casos os quais determinam a inclusão ou exclusão de informações. Utilizou-se a Taxonomia II da NANDA-I (2010) para redação dos Diagnósticos de Enfermagem e o raciocínio diagnóstico segundo modelo de Risner $^{(8)}$.

Com os casos clínicos prontos e seus respectivos quadros de raciocínio diagnóstico e diagnósticos de enfermagem elaborados, passou-se à validação das evidências com enfermeiras expertas (quarto passo) e construção dos roteiros de orientação (quinto passo).

O sexto passo refere-se a obter a validade do conteúdo e a realizar alterações, se necessário. A validade foi verificada por enfermeiras expertas com graus avançados de conhecimento na temática.

\section{RESULTADOS}

Apresenta-se primeiramente, os resultados relativos à coleta de casos e após, os passos para construção e validação de estudos de casos com finalidade educacional.

\section{Coleta dos casos}

Inicialmente realizou-se a discussão prévia para adequação de horários e da coleta de dados na UCIN. Oito prematuros foram avaliados pela pesquisadora e imediatamente após pela enfermeira experta; a seguir cada uma delas registrava em formulário próprio os Diagnósticos de Enfermagem utilizando o modelo de raciocínio de Risner.

Ao realizar a comparação dos dados obtidos pelas duas enfermeiras, obteve-se concordância máxima de $75 \%$, mas observou-se que a realização do exame físico uma após a outra ocasionava alteração dos dados na segunda coleta devido ao manejo prévio do prematuro. Assim, estabeleceu-se a realização do exame físico no mesmo momento; a inspeção do prematuro passou a ser realizada por uma avaliadora enquanto a outra observava e, se julgasse necessário, também fazia o manejo do bebê, excetuando-se a ausculta e apalpação que foram realizadas pelas duas avaliadoras.

A partir daí, realizou-se a avaliação clínica de mais sete RNPT e foi obtida a média de $92,5 \%$ de concordância entre os avaliadores, a qual foi considerada adequada, em todas as etapas (exame físico, quadro de raciocínio diagnóstico de Risner e estabelecimento dos diagnósticos de enfermagem segundo taxonomia NANDA-I 2010).

Após conclusão dessa etapa de calibração, a pesquisadora realizou a coleta e adequação de sete casos que foram incluídos finalmente adaptados e validados para o ensino de enfermagem.

Coletou-se os casos clínicos de RNPT com base no instrumento de coleta de dados elaborado a partir de um roteiro já utilizado na prática clínica da UCIN, no qual constam os seguintes dados: oxigenação (tipo e características da respiração, frequência respiratória, ausculta pulmonar, saturação de oxigênio), circulação (ausculta cardíaca, frequência cardíaca, pulsos, rede vascular), termorregulação (temperatura axilar, temperatura cutânea, incubadora, uso de roupas), alimentação (tipo, volume, características do abdômen, fígado, sonda gástrica ou enteral, amamentação), eliminação (características e volume de urina e evacuação, secreção gástrica e ostomias), integridade tecidual (características da pele, feridas, acesso venoso, tipos de infusão venosa, fototerapia), mobilidade (tônus muscular, posição, mobilidade), sono e repouso (padrão de sono, manipulação, características do ambiente quanto à luminosidade e ao ruído), percepção sensorial (reflexos, avaliação da dor), comunicação e interação (relacionamento familiar, contato pele a pele) e análise de prontuário.

Após a coleta dos casos clínicos utilizou-se as recomendações ${ }^{(4)}$ para elaboração de estudos de casos com finalidades educacionais, descritas abaixo.

\section{Primeiro passo: seleção de recursos e métodos}

Atendidos os critérios de inclusão, selecionaram-se sete casos reais de RNPT internados na UCIN do hospital. A seleção foi por conveniência buscando condições diferenciadas entre os prematuros, mas frequentes nas unidades neonatais, na tentativa de representar as situações clínicas e os diagnósticos de enfermagem mais comuns.

Foi realizado o exame físico, seguindo as necessidades humanas básicas, e análise de prontuário em buscas de evidências. Os casos foram coletados em folha de papel, digitado em programa MSWord 2003 e 
posteriormente adaptados para que refletissem as situações clínicas comuns da atenção ao RNPT.

\section{Segundo passo: objetivos dos casos}

Os objetivos estabelecidos para a utilização dos casos foram:

$\checkmark$ Representar situações típicas de RNPT em UCIN, favorecendo $\mathrm{o}$ aprendizado do raciocínio diagnóstico pelos alunos de enfermagem e enfermeiros;

$\checkmark$ Descrever o cuidado em enfermagem ao recémnascido de forma integral, demonstrando as relações entre as necessidades humanas básicas;

$\checkmark$ Descrever os casos de forma clara e compreensível, utilizando linguagem padronizada com a prática de enfermagem.

\section{Terceiro passo: complexidade dos casos}

Os dados coletados foram digitados em texto cursivo e distribuídos em "antecedentes", "avaliação clínica", "dados complementares" e "dados da mãe/família", compreendendo as necessidades psicobiológicas e psicossociais do RNPT e família.

Elegeu-se os seguintes Diagnósticos de Enfermagem para inclusão nos casos: Atraso no Crescimento e no Desenvolvimento, Dor Aguda, Integridade da Pele Prejudicada, Padrão Ineficaz de Alimentação do Bebê, Proteção Ineficaz, Risco de Aspiração, Risco de Desequilíbrio na Temperatura Corporal, Risco de Infecção, Risco de Integridade da Pele Prejudicada, Risco de Vínculo Prejudicado, Termorregulação Ineficaz, Amamentação Ineficaz, Disposição para Aumento da Competência Organizacional do Bebê, Comportamento Desorganizado do Bebê, Risco de Comportamento Desorganizado do Bebê, Risco de Crescimento Desproporcional, Risco de Atraso no Desenvolvimento, Maternidade Prejudicada e Risco de Volume de Líquidos Deficiente.

Após a formatação dos dados obteve-se os seguintes estudos de caso:

$\checkmark$ Jaime: idade gestacional de $28 \mathrm{~s} 4 \mathrm{~d}$, idade corrigida 39s3d e $14 \mathrm{DE}$.

$\checkmark$ Sofia: idade gestacional de 32s5d, idade corrigida 35s3d e 12 DE.

$\checkmark$ Lorena: idade gestacional de $36 s 1 d$, idade corrigida $36 \mathrm{~s} 3 \mathrm{~d}$ e $06 \mathrm{DE}$.

$\checkmark \quad$ José: idade gestacional de 27s3d, idade corrigida 32s4d e 13 DE.

$\checkmark$ João: idade gestacional de 32s1d, idade corrigida 33s4d e $10 \mathrm{DE}$. $\checkmark \quad$ Maria: idade gestacional de 33s, idade corrigida 36s1d e $8 \mathrm{DE}$.

$\checkmark \quad$ Ana: idade gestacional de 36s1d, idade corrigida 36s6d e 9 DE.

Assim, foram incluídos 72 diagnósticos para o ensino do raciocínio diagnóstico, entretanto há diagnósticos repetidos, pois muitos são comuns à situação de prematuridade e hospitalização em UCIN.

\section{Quarto passo: validação das evidências}

Para realizar a validação das evidências adaptadas nos casos, foram convidadas duas enfermeiras pesquisadoras que atuam no ensino de graduação e pósgraduação em enfermagem bem como na pesquisa e extensão de serviços à comunidade.

Uma delas tem ampla experiência prática, de ensino em pesquisa na temática raciocínio diagnóstico e processo de enfermagem, a qual atingiu 12 pontos no instrumento de pontuação para seleção de expertos adaptado(16). A outra atua também no ensino, pesquisa e extensão, na temática de cuidado ao RNPT em unidades neonatais e somou 10 pontos no instrumento de pontuação para seleção de expertos adaptado(16).

Foram realizadas reuniões (enfermeiras-docentes e pesquisadora) para discussão grupal e validação das evidências dos casos e da terminologia utilizada, perfazendo 24 horas de trabalho.

Para discussão e elaboração do raciocínio diagnóstico utilizou-se conhecimento prático e teórico, livros textos de referência para o cuidado ao RNPT e família, além de artigos científicos, dissertações e teses.

\section{Quinto passo: roteiros de orientação}

Foi construído roteiro para auxiliar o estudante ou enfermeiro a utilizar os estudos de caso.

\section{Sexto passo: validade do conteúdo}

A validade de conteúdo seguiu os passos executados na validação das evidências (quarto passo).

Foram convidadas duas enfermeiras pesquisadoras que atuam no ensino de graduação de enfermagem ministrando conteúdos sobre raciocínio diagnóstico em enfermagem e três outras que atuam no ensino sobre os cuidados de enfermagem ao recém-nascido pré-termo e família em unidades neonatais. Todas somaram 12 pontos no instrumento de pontuação para seleção de expertos $\operatorname{adaptado}^{(16)}$.

O grupo se reuniu em 14 encontros para os sete casos, perfazendo um total de 56 horas de trabalho para padronizar a redação cursiva dos casos e avaliar os 
raciocínios diagnósticos elaborados previamente pela pesquisadora.
O Quadro 2 sumariza os passos realizados para elaboração e validação de casos com finalidade educacional.

Quadro 2: Descrição de atividades realizadas para elaboração e validação de casos com finalidade educacional de acordo com referencial teórico adotado. Ribeirão Preto, SP, Brasil, 2010.

\begin{tabular}{|c|c|c|}
\hline Passo & Objetivo & Atividades realizadas \\
\hline \multirow{3}{*}{ Passo 1} & \multirow{3}{*}{ Seleção de recursos e métodos } & Seleção do local de coleta \\
\hline & & Seleção de instrumento de coleta de dados \\
\hline & & Seleção de recursos materiais, físicos e humanos \\
\hline \multirow{3}{*}{ Passo 2} & \multirow{3}{*}{ Estabelecimento dos objetivos } & Ensino \\
\hline & & Assistência \\
\hline & & Qualidade técnica do caso \\
\hline \multirow{3}{*}{ Passo 3} & \multirow{3}{*}{ Complexidade dos casos } & Coleta de acordo com as necessidades humanas básicas \\
\hline & & Utilização do modelo de raciocínio diagnóstico de Risner \\
\hline & & $\begin{array}{c}\text { Utilização da taxonomia II da NANDA-I (2010) para redação dos } \\
\text { Diagnósticos de Enfermagem }\end{array}$ \\
\hline \multirow{3}{*}{ Passo 4} & \multirow{3}{*}{ Validação das evidências } & Estabelecimento de critérios para seleção de enfermeiras expertas \\
\hline & & Organização, discussão das evidências \\
\hline & & $\begin{array}{l}\text { Adequação das terminologias pertinentes à área da saúde e de } \\
\text { enfermagem }\end{array}$ \\
\hline Passo 5 & Roteiros de orientação & Elaboração de roteiros para utilização dos casos \\
\hline \multirow{4}{*}{ Passo 6} & \multirow{4}{*}{$\begin{array}{l}\text { Validade do conteúdo e a } \\
\text { realização de alterações }\end{array}$} & Estabelecimento de critérios para seleção de enfermeiras expertas \\
\hline & & $\begin{array}{c}\text { Organização do caso, do raciocínio diagnóstico e dos diagnósticos } \\
\text { de enfermagem }\end{array}$ \\
\hline & & Organização do trabalho grupal \\
\hline & & Adequação dos casos após validação \\
\hline
\end{tabular}

\section{DISCUSSÃO}

O estudo de caso enquanto estratégia de ensino pode ser uma ponte entre as lacunas da educação e da aquisição de habilidades clínicas para a progressão na profissional com vistas à segurança e qualidade da assistência ao paciente, família e comunidade ${ }^{(10)}$.

Estratégias de ensino pautadas no método ativo devem promover a articulação entre a teoria e a prática tanto para o ensino de graduação como para a educação permanente de profissionais de enfermagem ${ }^{(6-7)}$. Nesse sentido, os estudos de caso podem favorecer a problematização a qual implica na participação dos agentes na construção de seus conhecimentos, na troca de experiências e no questionamento sobre a sua realidade.

Durante a realização desse estudo observou-se que a opção pela construção de casos seguindo o referencial educacional permitiu a construção de casos bem elaborados e potencializou uma linguagem científica consistente e refinada para auxiliar o ensino do pensamento crítico.

Em outras áreas de conhecimento também se têm adotado referenciais teóricos para a elaboração de casos. $\mathrm{Na}$ área da administração(7) e medicina(20) os modelos adotados apresentam passos semelhantes ao utilizado nesse estudo no que se refere ao estabelecimento de objetivos, definição de complexidade de informações, precisão de conteúdo de acordo com a realidade e o detalhamento necessário para a compreensão do caso.

No entanto, para o ensino em enfermagem há poucos guias/instrumentos/modelos específicos para a construção de casos.

Nesse aspecto estudo realizado no ensino da prática avançada em enfermagem neonatal(21) apresentou uma abordagem de ensino baseada no uso de estudo de caso adaptado. Segundo estudo, o caso deve conter instruções gerais para sua operabilidade, estar relacionado com o tema e centrado nos objetivos do curso.

Estudos de caso adequados para fins educacionais podem simular a realidade prática e aproximar o estudante ou enfermeiro da realidade vivenciada na clínica, estimulando-os no processo de aprendizagem.

Teoria de aprendizagem ${ }^{(22)}$ sobre o ensino de adultos têm demonstrado que, para eles aprenderem, faz-se necessário que o professor se utilize de situações reais, focadas no aluno, tornando-o ativo no processo de ensinoaprendizagem.

Os casos elaborados nesse estudo foram desenvolvidos para atender a recomendação de problematizar as situações e buscar a inserção crítica dos participantes na sua realidade. Para tanto se utilizou problemas existentes nas experiências cotidianas do aprendiz, os quais foram sistematizados e teorizados tendo em vista a relação dialógica e participativa, 
conduzindo-o à reflexão(6-7) e ação na perspectiva da construção de competência clínica e de vivência de uma prática reflexiva.

Estudos realizados na África do Sul(22) e nos Estados Unidos $^{(10)}$ demonstrou que casos adaptados foram bem aceitos por professores ${ }^{(24)}$ e alunos ${ }^{(10)}$ podendo impactar positivamente no ensino de enfermagem e na preparação para a prática profissional.

Em contrapartida, pesquisa(9) realizada com 439 alunos de medicina do último ano em uma universidade americana, divididos em grupo controle e experimental, demonstrou que o estudo de caso quando aplicado isolado enquanto estratégia de ensino pode não favorecer o estímulo do pensamento crítico entre estudantes.

Os casos devem estar de acordo com o objetivo do curso e proporcionar ao estudante experiências para desenvolver as competências cognitivas, comunicacionais e procedimentais ${ }^{(1,3,6,10,13)}$ a serem desenvolvidas na tentativa de prover oportunidades frequentes de reforço positivo.

Apesar de recomendação para a utilização de poucas informações na elaboração de $\operatorname{casos}^{(7)}$, de acordo com o terceiro passo do referencial adotado(4) $^{(4) z-s e ~ a ~ o p c ̧ a ̃ o ~ p o r ~}$ dados que agregassem complexidade aos estudos de caso. As associações de várias informações sobre o estado clínico, dados laboratoriais, situações sociais e familiares do RNPT têm sido considerado um paradigma na construção de casos, mas entende-se que tal atitude estimula a aprendizagem do cuidado integral(1). Nesse sentido, é papel do professor selecionar o material de estudo e as categorias que devem compor a situação a ser simulada nos estudos de caso ${ }^{(6)}$.

Outro aspecto referente a análise dos dados utilizados nos casos clínicos e também sobre os casos prontos para o ensino, evidenciou que nenhum dos referenciais citados $^{(1,7)}$ para construção de casos fizeram discussão sobre questões relacionadas a validação conforme referencial adotado nesse estudo.

Não foram localizados estudos que se dedicaram a debater a validação de evidências e de casos para o ensino em saúde ou enfermagem denotando lacuna de conhecimento.

Estudos de caso que reflitam a realidade e permitam a aprendizagem são difíceis de serem desenvolvidos, pois eles são utilizados como ferramentas para mensurar fenômenos de enfermagem ${ }^{(4,15)}$.

Para desenvolver bons estudos de caso, minimamente os princípios de medição (validade confiabilidade) devem ser realizados ${ }^{(4,15)}$.
Cabe ressaltar que a elaboração e validação de estudos de caso no ensino de enfermagem, especialmente enfermagem neonatal, voltados para a aprendizagem participativa ainda necessita de mais pesquisas tanto no ponto de vista pedagógico quanto metodológico.

Além disso, a inserção de diferentes formas de ensinar está longe de se esgotar. Os avanços diários no processo de aprendizagem podem contribuir para a educação superior em enfermagem aproximando-a do seu contexto social, político e econômico.

\section{CONSIDERAÇÕES FINAIS}

A utilização de estudos de caso no ensino do pensamento crítico e raciocínio diagnóstico em enfermagem neonatal são possibilidades que podem ser contempladas, entretanto, devem ser guiados pelas boas práticas na educação, garantindo motivação, desempenho e satisfação de estudantes e professores.

As etapas de elaboração e de validação dos casos seguiram metodologia rigorosa por acreditar-se que casos bem estruturados podem se tornar recurso importante na aprendizagem dos estudantes de enfermagem e enfermeiros.

Os passos para desenvolvimento dos casos explicitados nesse estudo merecem destaque pela seriedade dos profissionais envolvidos no compromisso de adequar casos clínicos reais que refletissem a tomada de decisão clínica no contexto da aprendizagem.

Outro aspecto relevante quanto aos estudos de caso refere-se à validação deles para o ensino em saúde ou enfermagem, o qual ainda é uma lacuna de conhecimento.

Há também a necessidade de avaliação da aprendizagem e satisfação de alunos e professores do ensino superior em enfermagem, mediados pelos estudos de caso que foram desenvolvidos e validados.

Recomenda-se o uso de estudos de casos baseados em situações reais vivenciadas durante as atividades de ensino na tentativa de transpor a lacuna entre o ensino teórico e prático.

\section{FINANCIAMENTO}

Conselho Nacional de Desenvolvimento Científico e Tecnológico - CNPq, Edital MCT/CNPq N 014/2008 Universal - Processo no 473217/2008-2. 


\section{REFERÊNCIAS}

1. Bosque E. An application of integrative, case-based, nursing education for neonatal advanced practice. Adv Neonatal Care. [Internet]. 2012 [acesso em: 20 nov 2013]; 12(5): 292-302. Disponível em:

http://dx.doi.org/10.1097/ANC.0b013e318262499b.

2. Alfaro-Lefevre R. Aplicação do processo de enfermagem: promoção do cuidado colaborativo. Porto Alegre: Artmed; 2010.

3. Cates LA. Simulation Training: a multidisciplinar approach. Adv Neonatal Care. [Internet]. 2011 [acesso em: 20 nov 2013];

11(2): 95-100. Disponível em:

http://dx.doi.org/10.1097/ANC.0b013e318210d16b.

4. Lunney M. Pensamento Crítico para o Alcance de Resultados

Positivos em Saúde: Análises e Estudos de Caso em

Enfermagem. Porto Alegre: Artmed; 2010.

5. Duarte APP, Ellensohn L. A operacionalização do processo de enfermagem em terapia intensiva neonatal. Revista enfermagem UERJ. [Internet]. 2007 [acesso em: 20 ago 2012]; 15(4):521-6. Disponível em: http://www.facenf.uerj.br/v15n4/v15n4a07.pdf. 6. Anastasiou LGC, Alves LP. Estratégias de Aprendizagem. In: Anastasiou LGC, Alves LP. Processos de ensinagem na universidade: pressupostos para as estratégias de trabalho em aula. 10a ed. Joinville: Univille; 2012. p. 91.

7. Graham A. Como escrever e usar estudos de caso para ensino e aprendizagem no setor público. Brasília: ENAP, 2010, 60p.

8. Risner PB. Diagnosis: analysis and synthesis of date. In: Christensen, P. J.; Kenney, J. W. Nursing process: application of conceptual models. St. Louis: Mosby; 1990.

9. Abraham R, Ramnarayan K, Kamath A. Validating the effectiveness of Clinically Oriented Physiology Teaching (COPT) in undergraduate physiology curriculum. BMC Medical Education. [Internet]. 2008 [acesso em: 20 nov 2013]; 8:40. Disponível em: http://dx.doi.org/10.1186/1472-6920-8-40.

10. Cates LA, Armentrout D. Simulation with synergy and community energy. Adv Neonatal Care. [Internet]. 2013 Apr[acesso em: 20 nov 2013]; 13(2):97-102. Disponível em: http://dx.doi.org/10.1097/ANC.0b013e318285fb82.

11. Zimmerman D, Pilcher J. Implementing NICU critical thinking programs: on unit's experience. Neonatal network. $2008 \mathrm{Jul}$; 27(4): 231-238.

12. Cates LA, Wilson D. Acquisition and maintenance of competencies through simulation for neonatal nurse practitioners. Adv Neonatal Care. [Internet]. 2011 [acesso em: 20 nov 2013]; 11(5): 321-327. Disponível em:

http://dx.doi.org/10.1097/ANC.0b013e31822a34a0.

13. Arafeh JMR. Simulation-based training: the future of competency? J Perinat Neonat Nurs. [Internet]. 2011 25(2):

171-174. Disponível em: http://dx.doi.org/

10.1097/JPN.0b013e3182116e55.

14. Gallo AM. Using technology to meet the educational needs of multigenerational perinatal nurses. J Perinat Neonat Nurs.

[Internet]. 2011 25(2): 195-199. Disponível em:

http://dx.doi.org/10.1097/JPN.0b013e3182163993.

15. Lunney M. Current Knowledge Related to Intelligence and

Thinking With Implications for the Development and Use of Case

Studies. International Journal of Nursing Terminologies and

Classifications. [Internet]. 2008 oct; 19(4): 158-162. Disponível

em: http://dx.doi.org/10.1111/j.1744-618X.2008.00104.x.

16. Góes FSN, Fonseca LMM, Furtado MCC, Leite AM, Scochi CGS. valuation of the virtual learning object "Diagnostic reasoning in nursing applied to preterm newborns". Rev. LatinoAm. Enfermagem. [Internet]. 2011 Aug [acesso em: 15 ago 2012]; 19( 4 ): 894-901. Disponível em:

http://dx.doi.org/10.1590/S0104-11692011000400007.

17. Carmona EV, Lopes MHBM. Content validation of parental role conflict in the neonatal intensive care unit. Int J Nurs Terminol Classif 2006; 17(1);3-9.

18. Fehring RJ. Validating diagnostic labels: standard methodology nursing diagnosis. In: Hurley $M$, editor. Classification of nursing diagnoses: proceedings of the sixth conference. St. Louis: Mosby; 1986.

19. Pileggi SO. Validação clínica do diagnóstico de enfermagem desobstrução ineficaz de vias aéreas de crianças e adolescentes submetidos à correção cirúrgica de cardiopatia congênita [dissertação]. Ribeirão Preto: Escola de Enfermagem de Ribeirão Preto/USP; 2007. 224p. Disponível em: http://www.teses.usp.br/teses/disponiveis/22/22132/tde05102007-173932/pt-br.php.

20. Sebastiani RL, Zago MK, Mantovani A, Bez MR, Bruno R, Dahmer A, Flores CD. Ferramenta de autoria para construção de casos clínicos interativos para educação médica. Disponível em: http://www.imago.ufpr.br/csbc2012/anais csbc/eventos/wim/art igos/WIM2012\%20-

\%20Ferramenta $\% 20$ de $\% 20$ Autoria $\% 20$ para $\% 20$ Construcao $\% 20$ de $\% 20$ Casos $\% 20$ Clinicos $\% 20$ Interativos $\% 20$ para\%20Educacao $\% 20$ Medica.pdf.

21. Dewey J. The educational situation: as concerns the elementary school. Curriculum Studies. 2001; 33(4), 387-403. 22. Maunye TJ, Meyer SM, Van Velden CE. An assessment of teaching strategies used by lecturers at a nursing college in Mpumalanga. Curationis. [Internet]. 2009 Sep [acesso em: 15 ago 2012]; 32(3): 30-7. Disponível em: http://dx.doi.org/ 10.4102 /curationis.v32i3.1221.

Artigo recebido em 08/10/12.

Aprovado para publicação em 06/11/13.

Artigo publicado em 31/03/2014. 